\title{
MUDAS E MASSAS CORPORAIS EM AVES MIGRATÓRIAS DO MANGUE DA CARAPEBA, RIO DE JANEIRO, BRASIL
}

\author{
JULIANA YWASAKI LIMA ${ }^{1}$, RONALDO NOVELLI ${ }^{2}$, CARLOS EURICO PIRES FERREIRA TRAVASSOS ${ }^{3}$ \\ ${ }^{1}$ Universidade Estadual do Norte Fluminense Darcy Ribeiro, Centro de Tecnologias Agropecuáriasl. (e-mail: ju.ywasaki@gmail.com) \\ ${ }^{2,3}$ Universidade Estadual do Norte Fluminense Darcy Ribeiro, Centro de Biociências e Biotecnologia, Lca. Av Alberto Lamego, 2000 \\ Horto. 28015-620 - Campos dos Goytacazes, RJ - Brasil.
}

\section{RESUMO}

Nas aves migratórias o estágio do ciclo de vida e a atividade de muda parecem ser influenciados pela massa corporal que varia com estação, hora do dia, sexo e entre populações. Estas variações verificadas na muda em diversas localidades também podem estar relacionadas ao tempo de permanência na área e à oferta e qualidade alimentar local, bem como às variações geográficas das espécies. O estudo objetivou comparar a massa corporal das aves migratórias do Mangue da Carapeba, Campos dos Goytacazes-Rio de Janeiro com a região Norte do Brasil. Os dados foram coletados entre dezembro de 2006 a maio de 2009, a partir de 13 expedições a campo, realizadas no período de verão (menos junho e julho, que as aves estão migrando) e período de inverno. Foram utilizadas 12 redes ornitológicas de $12 \mathrm{~m}$ x $3 \mathrm{~m}$ e $36 \mathrm{~mm}$ de malha. A média das massas corporais observadas no Mangue da Carapeba, para oito das espécies estudadas, foi menor que no Norte do Brasil. $\mathrm{Na}$ análise das mudas os dados para Charadrius semipalmatus são similares a região Norte brasileira. Em Actitis macularius não houve padrão bem definido na distribuição das mudas das rêmiges. Poucos indivíduos apresentaram muda nas retrizes, dificultando a avaliação. Do mesmo modo, padrões para muda das rêmiges de Calidris fuscicolis, Numenius phaeopus e Pluvialis squatarola, não foram detectadas devido ao tamanho reduzido da amostra. A amostragem baixa de aves com muda nas retrizes não permitiu detectar nenhum padrão. As variações encontradas nas mudas e na massa corporal podem estar relacionadas à época, oferta e qualidade alimentar das diferentes áreas e às variações geográficas das espécies.

PALAVRAS-CHAVE: Avifauna, Charadriiformes, Migração, Norte Fluminense.

\section{ABSTRACT}

In migratory shorebirds the stage of the lifecycle and feather moultappear to be influenced by body mass, which varies with season, time of day, gender and among populations. These variations in moult in several locations also may be related to time spent in the area and the quality of local food supplies, as well as geographical variations of species. The study aimed to compare the body mass of migratory birds at the mangrove of Carapeba, Campos dos Goytacazes-Rio de Janeiro state, with those from the northern region of Brazil. Data were collected from December 2006 to May 2009, from 13 field expeditions, undertaken in the summer period (less June and July, the birds are migrating) and winter period. 12 of 12 mist nets were used, with $3 \mathrm{~m}$ and $36 \mathrm{~mm}$ of mesh. The average body mass observed at Carapeba mangrove was lower than at northern Brazil. The moult data of Semipalmated plover, Charadrius semipalmatus, was similar to those from North Brazil. A low number of birds was moulting tail feathers, so no clear pattern was detected. It was not possible to detect a patter in wing feather (remiges) moult of Spotted sandpiper, Actitis macularius, had. Similarly, patterns for wing feather moult of White-rumped sandpiper, Calidris fuscicolis, Whimbrel Numenius phaeopus, and Black-bellied plover Pluvialis squatarola, were not detected due to low sample size. Variations found in feather moult schedule and body mass may be related to availability of and quality of food supply in different areas, as well as geographical variations of the species.

KEYWORDS: Avifauna, Charadriiformes, Migration, North Fluminense.

\section{INTRODUÇÃO}

Anualmente chegam ao Brasil milhões de aves migratórias de várias espécies. Muitas realizam migrações sazonais da América do Norte para a América do Sul e vice-versa (Sick 1997; Morrison et al. 1989).

Atraídas pela abundante oferta de alimento, percorrem longas distâncias e permanecem parte do ano em território brasileiro (Andrade 1997; Sick 1983; Azevedo Jr. et al. 2001a,b). Muitas espécies são fiéis aos sítios de invernada, retornando ao mesmo local todos os anos (Castro \& Myers 1987; Azevedo Jr. et al. 2001a,b; 2002). Batuíras (Charadriidae) e maçaricos (Scolopacidae), aglomeram-se aos milhares todos os anos nas áreas com abundância de recursos alimentares (Azevedo Jr. et al. 2001b).

A identificação dos sítios de importância para aves aquáticas é uma ferramenta valiosa na conservação da biodiversidade (Blanco 1999), onde alterações nos padrões das populações podem servir de indicador da qualidade ambiental (Goldsmith 1991; Blanco 1999).

As aves migratórias aquáticas encontram na região da Bacia de Campos um refúgio importante, não apenas nas lagoas costeiras associadas às restingas, como também mais para o interior (Alves et al. 2009). Diversas espécies de aves migratórias, incluindo migrantes neárticas de longa distância, utilizam as restingas durante uma época do ano, assim como visitantes que nelas ocorrem durante uma parte do ano (Alves 2007).

Muitas áreas de alimentação e descanso ainda são desconhecidas, como é o caso do Mangue da Carapeba, localizado no município de Campos dos Goytacazes-Rio de Janeiro. De acordo com Bianchi 
2007; Nacinovic, J. 2005; Santos, T.R.S. \& Alves, M.A.S. 2011, a região Norte Fluminense possui excelentes sítios de invernada para as aves migratórias oriundas da América do Norte, sendo dessa forma, um bom lugar para o monitoramento de várias espécies.

Estudos utilizando a marcação de aves para obtenção de dados sobre a biologia, longevidade e rotas de espécies migratórias são importantes e vêm contribuindo para a conservação destas em todo o mundo (Tavares \& Siciliano 2014). Medidas morfométricas são informações básicas acerca da biologia das aves, e possuem um importante papel em estudos sobre os padrões de variação geográfica das espécies (Karr 1979). Dados sobre mudas e parâmetros biométricos são comuns em Passeriformes (Cavalcanti \& Marini 1993) e alguns autores realizaram estudos sobre várias espécies do mundo, tema no qual se destaca o trabalho de Dunning 2008. Em relação às aves migratórias, dados de muda e parâmetros biométricos podem ser encontrados em Gaston \& Blackburn (1995), Nascimento (1998), Azevedo Júnior \& Larrazábal (1999), Azevedo Jr et al. (2001a), Schulz-Neto et al. (2008), Legagneux et al. (2012).

Geralmente observam-se duas mudas nas aves, uma antes da reprodução e outra após, podendo ocorrer variações entre espécies e entre indivíduos da mesma espécie. Em maçaricos e batuíras ocorrem as mudas para a reprodução e no período pós-reprodutivo, com a troca simultaneamente das penas da asa e cauda (Azevedo Jr et al. (2001a).

Clark-Jr (1979) afirma que a massa corporal das aves varia de acordo com a estação, à hora do dia, o sexo e entre as populações. O estágio do ciclo de vida e a atividade de muda, principalmente no caso das aves migratórias, também parecem influenciar, pois a energia despendida durante a reprodução, o descanso reprodutivo e a troca de penas é diferente e exige diferentes esforços fisiológicos (Gill 1995). Nas espécies migratórias, o considerável desgaste das penas confere ao fenômeno da muda conotação especial, em função da demanda energética envolvida e da influência que exerce na capacidade de vôo (Nascimento 1998). Estas variações verificadas no processo de muda em diversas localidades também podem estar relacionadas ao tempo de permanência em cada área, a oferta e qualidade alimentar das diferentes áreas, uma vez que o gasto energético durante a muda também é elevado, bem como a variações geográficas das espécies (Schulz-Neto et al. 2008).

Adultos começam a muda perto do fim da atividade reprodutora, enquanto os imaturos começam a muda mais cedo (Novelli 1997). Em maçaricos geralmente as retrizes aparecem primeiro, sendo as rêmiges mudadas mais tarde, especialmente nas aves que fazem longos voos ininterruptos. No final do inverno ou primavera, a plumagem de inverno é mantida e em alguns também as retrizes (Matthienssen 1967).

Este estudo teve como objetivo fazer uma comparação da massa corporal das aves migratórias ocorrentes no Mangue da Carapeba, município de Campos dos Goytacazes-RJ com a região Norte do Brasil e também analisar o padrão de mudas nas espécies.

\section{MATERIAL E MÉTODOS}

As capturas das aves foram realizadas em uma área de planície costeira da região Norte Fluminense, especificamente no Mangue da Carapeba, situada no distrito Farol de São Tomé, município de Campos dos Goytacazes, entre as coordenadas geográficas $22^{\circ} 4^{\prime} 57,59$ "S e $41^{\circ} 7^{\prime} 20,25^{\prime \prime W}$.

Para a análise das mudas e massa corporal os dados foram coletados no período de dezembro de 2006 a maio de 2009, a partir de 13 expedições a campo, realizadas no período de verão (menos junho e julho, que as aves estão migrando) e período de inverno. Foram utilizadas 12 redes ornitológicas de 12 $\mathrm{m} \times 3 \mathrm{~m}$ e $36 \mathrm{~mm}$ de malha, perfazendo um total de $432 \mathrm{~m}$ de redes e fixadas com base em observações preliminares da movimentação das aves na área de estudo. Estas eram abertas às $18 \mathrm{~h}$ e fechadas às $6 \mathrm{~h}$, somando um esforço de captura de $432 \mathrm{~m}^{2} / 12 \mathrm{~h}$ de redes por amostragem.

Para a obtenção da massa corporal foram utilizadas balanças (Pesolas ${ }^{\circledR}$ ) que variavam de $100 \mathrm{~g}$ a $1000 \mathrm{~g}$. Utilizou-se uma ficha específica, onde anotou-se dados biológicos referentes à data, espécie, idade, muda (rêmiges e retrizes), massa corporal total e número da anilha. Foi aplicado teste 
não paramétrico $U$ de Mann-Whitney nas espécies com mais de dez exemplares para verificar diferença significativa entre as variáveis de média do presente estudo em relação as variáveis do Norte do Brasil.

$\mathrm{Na}$ análise das mudas, as aves foram analisadas quanto às rêmiges primárias e retrizes. Para o anilhamento foram seguidas as normas internacionais, $e$ as informações repassadas ao CEMAVE/IBAMA.

\section{RESULTADOS E DISCUSSÃO}

Foram capturados 290 indivíduos, pertencentes a 2 famílias e 8 espécies, sendo todas migratórias, seguindo critérios do CBRO (listas das aves do Brasil, 2014). A lista das espécies e o número de exemplares analisados é apresentada na tabela 1.

TABELA 1 - Lista das espécies e o número de exemplares analisados, capturados no Mangue da Carapeba, Campos dos Goytacazes, Rio de Janeiro, no período de dezembro de 2006 a maio de 2009.

\begin{tabular}{lc}
\hline ESPÉCIES & NÚMERO DE INDIVÍDUOS \\
\hline Actitis macularius & 24 \\
Arenaria interpres & 88 \\
Calidris fuscicollis & 8 \\
Charadrius collaris & 63 \\
Charadrius semipalmatus & 86 \\
Numenius phaeopus & 9 \\
Pluvialis squatarola & 6 \\
Tringa melanoleuca & 6 \\
\hline TOTAL & 290 \\
\hline
\end{tabular}

\section{Massa corporal}

Dados de massa corporal têm sido frequentemente utilizados como índices para acompanhar o processo migratório (Mascher 1966) e é considerada a melhor medida para caracterizar o tamanho da ave (Dunning 1993). A massa corporal de algumas das espécies mais relevantes deste estudo foi comparada com as médias obtidas por SchulzNeto et al. (2008) na tabela 2. Foram considerados as mesmas épocas em ambos os estudos, comparandose massas corporais no período de verão (menos junho e julho, que as aves estão migrando) e período de inverno.

TABELA 2 - Comparação de massas corporais das espécies $(n=7)$ obtidas neste estudo com as apresentadas em Schulz-Neto et al. (2008). Média (mínimo-máximo).

\begin{tabular}{lllll}
\hline Espécies & $\begin{array}{l}\text { Número } \\
\text { Amostral }\end{array}$ & Idade & Presente Estudo & $\begin{array}{l}\text { Schulz-Neto et al. } \\
\mathbf{( 2 0 0 8 )} \mathbf{( g )}\end{array}$ \\
\hline Actitis macularius & 24 & Adulto & $30,7(19-42)$ & $36,6(22-62)$ \\
\hline Arenaria interpres & 88 & Adulto & $81,2(67-106)$ & $92,1(57-127)$ \\
\hline Calidris fuscicollis & 8 & Adulto & $22,8(19-31)$ & $48,4(45-55)$ \\
\hline Charadrius collaris & 63 & Adulto & $31,4(23-49)$ & $29,2(24-40)$ \\
\hline $\begin{array}{l}\text { Charadrius } \\
\text { semipalmatus }\end{array}$ & 86 & Adulto & $39,6(32-50)$ & $39,4(20-70)$ \\
\hline Pluvialis squatarola & 6 & Adulto & $175,5(157-210)$ & $173,8(164-186)$ \\
\hline Tringa melanoleuca & 6 & Adulto & $62(43-70)$ & $141,7(115-172)$ \\
\hline
\end{tabular}


As médias das massas corporais observadas não apresentaram diferenças estatisticamente significativas (G.L:4; $p>0,05)$ com as variáveis do estudo de Schulz-Neto et al. (2008). Apesar disto, a maioria das médias foram maiores para a região Norte. Essas diferenças podem estar relacionadas aos diferentes estágios de ganho de massa corporal durante a migração, diretamente influenciado pelo tempo de permanência em cada área, ao estoque de gordura acumulado, à oferta e qualidade alimentar das diferentes áreas e as variações geográficas das espécies (Dunn et al. 1988).

\section{Mudas}

Analisando os dados de troca das rêmiges primárias verificou-se que a muda de inverno em $A$. interpres inicia no mês de setembro, trocando as penas mais internas 1 a 4 direita e esquerda. Já no mês de outubro apresentou mudas desde as mais internas 1 a 5 até as mais externas 6 a 8 . Mudas das penas 9 a 10 não foram observadas nos animais capturados, por tanto ocorreram nos meses subsequentes.

Para a espécie C. semipalmatus verificou-se que na captura de dezembro $100 \%$ dos indivíduos sem mudas (Figura 1). Isso demonstra que na amostragem deste mês, a espécie já havia completado sua muda de inverno. Alguns indivíduos capturados a posteriore estenderam suas mudas até fevereiro, trocando as penas mais externas 8 a10 direita e esquerda, finalizando a muda completa de inverno.

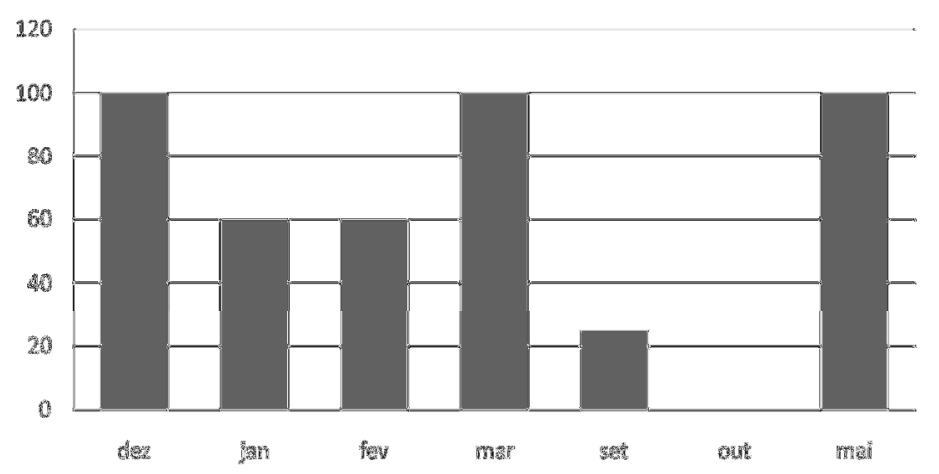

FIGURA 1 - Porcentagem de C. semipalmatus que não apresentaram muda nas rêmiges primárias. Capturados no Mangue da Carapeba, Campos dos Goytacazes, Rio de Janeiro, no período de dezembro de 2006 a maio de 2009.

Para C. collaris verificou-se que na captura de setembro $100 \%$ finalizaram sua muda (Figura 2). Entre dezembro e março alguns indivíduos capturados estenderam sua muda de verão (eclipse). Após o término do período reprodutivo todos os indivíduos já estavam com sua plumagem de inverno (maio).

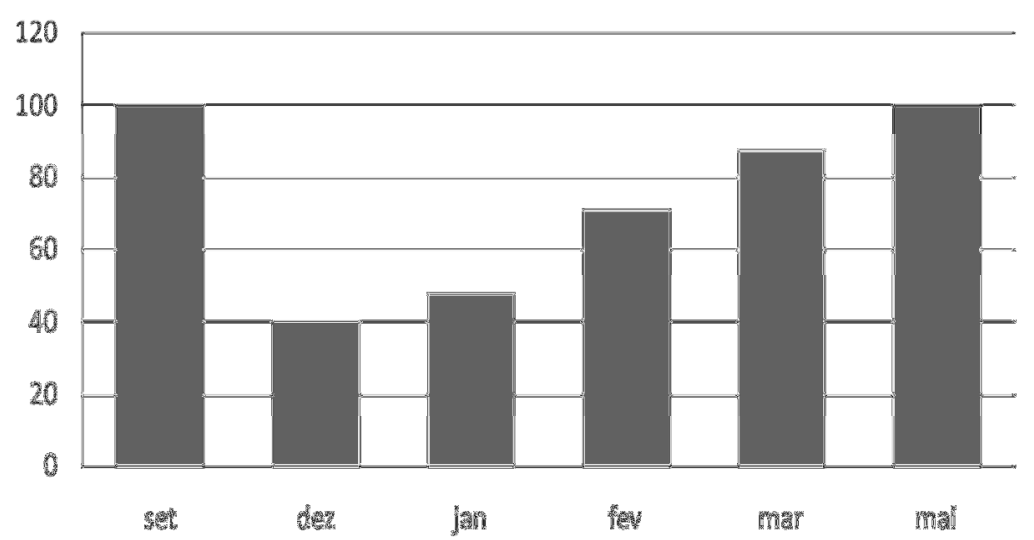

FIGURA 2 - Porcentagem de $C$. collaris que não apresentaram mudas nas rêmiges primárias. Capturados no Mangue da Carapeba, Campos dos Goytacazes, Rio de Janeiro, no período de dezembro de 2006 a maio de 2009. 
Os dados para a espécies $C$. semipalmatus concordam com os obtidos por Schulz-Neto et al. (2008) e Nascimento (1998). Em A. macularius não houve padrão bem definido na distribuição da muda das rêmiges. Para Calidris fuscicollis, $N$. phaeopus e $P$. squatarola não foi possível verificar um padrão de muda das rêmiges, pois o número de indivíduos que apresentaram mudas foi baixo. Em T. melanoleuca verificou-se que em dezembro havia mudas da pena 5 a 10, direita e esquerda, enquanto no mês de fevereiro um espécime apresentou muda nas penas mais externas (10 direita e esquerda), porém foi o único exemplar capturado fora do mês de dezembro, não sendo possível caracterizar um padrão de mudas para esta espécie.

$\mathrm{Na}$ análise de muda das retrizes poucos indivíduos apresentaram muda, não ocorrendo padrão bem definido para nenhuma das espécies estudadas. Para $A$. interpres, $C$. fuscicollis, $P$. squatarola e T. melanoleuca não foi possível analisar um padrão, pois o número de indivíduos capturados que apresentaram mudas foi baixo. Verificou-se em $C$. collaris que no mês de fevereiro estavam em muda as penas 1 a 5 , direita e esquerda. $C$. semipalmatus apresentou em fevereiro muda nas penas mais internas, i.e. 1-2 direita e esquerda, enquanto no mês de setembro apresentou muda nas penas mais externas 4 a 6 direita e esquerda. A. macularius apresentou em fevereiro muda nas penas 1 a 6 direita e esquerda e em janeiro apresentou muda somente na retriz 6 , porém foi um único espécime capturado que apresentou muda neste mês.

As mudas das penas da cauda, geralmente iniciam quando as primárias encontram-se na metade de sua muda (Karspryk \& Harrington 1989). A sequência na substituição das rectrizes pode ser variada (Ginn \& Melville 1995). As mudas de rectrizes em adultos de $C$. semipalmatus e jovens de $A$. interpres apresentaram uma sequência variada, concordando com Azevedo Jr. et al. (2001a).

Estas variações verificadas no processo de muda e não identificação de padrões de trocas das penas em diversas localidades (nesta região e no norte do país) também podem estar relacionadas ao tempo de permanência em cada área, a oferta e a qualidade alimentar das diferentes áreas, uma vez que o gasto energético durante a muda também é elevado.

\section{CONCLUSÕES}

Constataram-se variações na distribuição de mudas em diferentes períodos do ano e dificuldade na detecção de um padrão. A média das massas corporais encontradas nas espécies ocorrentes no Mangue da Carapeba não foi estatísticamente diferente da região norte do Brasil.

\section{REFERÊNCIAS BIBLIOGRÁFICAS}

ALVES, M.A.S.. 2007. Sistemas de migrações de aves em ambientes terrestres no Brasil: exemplos, lacunas e propostas para o avanço do conhecimento. Revista Brasileira de Ornitologia 15: 243-250.

ALVES, M.A.S.; JENKINS, C.N.; CARAMASCHI, E.P.; SCARANO, F.R.; OLIVEIRA, F.J.G.; ZALMON, I.R.; MONTEIRO, R.F.; CAMARGO A.F.; \& PIMM, S.L.. 2009. Região de Petróleo e gás Natural. In: BERGALLO H.G., FIDALGO E.C.C., ROCHA C.F.D., UZÊDA M.C., COSTA M.B., ALVES M.A.S., VAN SLUYS M., SANTOS M.A., COSTA T.C.C., COZZOLINO A.C.R. Estratégias e ações para a conservação da biodiversidade no estado do Rio de Janeiro. $1^{\text {a }}$ ed. instituto Biomas, v. 1, Rio de Janeiro. pp. 303-312.

ANDRADE, M.A.. 1997. A vida das aves: introdução à biologia e conservação. Belo Horizonte: Fundação Acangaú. 160p.

ANTAS, P.T.Z.. 1984. Migration of neartic shorebirds (Charadriidae and Scolopacidae) in Brazil - flyways and their different seasonal use. Wader Study Group Bull, 39:52-56.

AZEVEDO JÚNIOR, S.M. \& LARRAZÁBAL, M.E. 1999. Captura e anilhamento de Calidris pusilla (Scolopacidae) na costa de Pernambuco. Ararajuba, 7: 63-69.

AZEVEDO-JR., S.M.; DIAS, M.M.; LARRAZÁBAL, M.E.. 2001 a. Plumagens e mudas de Charadriiformes (aves) no litoral de Pernambuco, Brasil. Revista Brasileira de Zoologia, 18 (3):657672.

AZEVEDO-JR., S.M.; DIAS, M.M.; LARRAZÁBAL, M.E.; TELINO JR., W.R.; LYRANEVES, R.M.; FERNANDES, C.J.G..2001b. Recapturas e recuperações de aves migratórias no litoral de Pernambuco, Brasil. Ararajuba, 9 (1):33-42.

AZEVEDO JR., S.M.; DIAS, M.M.; LARRAZÁBAL, M. E.; FERNANDES, C. J. G.. 2002. Capacidade de vôo de quatro espécies de Charadriformes (aves) capturadas em Pernambuco, Brasil. Revista Brasileira de Zoologia, 19 (1): 183189.

BIANCHI, I.. 2007. Estudo do Vírus Influenza A em Aves Migratórias e Residentes do Norte do Estado do Rio de Janeiro, Brasil, no Período de 2004-2007. Universidade Estadual do Norte Fluminense, Campos dos Goytacazes (Tese de Doutorado).

BIERREGAARD JR., R.O.. 1988. Morphological data from understory birds in terra firme forest in the central Amazonian basin. Revista brasileira de Biologia, 48:169-178.

BLANCO, D.E.. 1999. Los humedales como habitat de aves acuaticas, p. 208-217. In: A.I. MALVÁREZ (Ed.). Tópicos sobre Humedales Subtropicales y Templados en Sudamérica. Montevideo, Orcyt-Unesco, 308p.

CASTRO, C. \& MYERS, J. P.. 1987. Ecología y conservación del 
playero Blanco (Calidris alba) en el Peru. Boletin Lima, p. 52, p. 47-61.

CAVALCANTI, R.B. \& MARINI, M.A.. 1993. Body masses of birds of the cerrado region, Brasil. Bulletin of the British Ornithologists' Club, 113:209-212.

CBRO- COMITÊ BRASILEIRO DE REGISTROS ORNITOLÓGICOS.. 2014. Listas das aves do Brasil. 11 Edição, 1/1/2014, Disponível em <http://www.cbro.org.br>. Acesso em: 20/02/2014.

CLARK-JR., G.A.. 1979. Body weights of birds: a review. Condor,81:193-202.

DUNN, P.O.; MAY, T.A.; MCCOLLOUGH, M.A. \& HOWE, M.A. 1988. Length of stay and fat content of migrant semipalmated sandpipers in Eastern Maine. Condor, 90:824-835.

DUNNING JR., J.B.. 2008. Handbook of avian body masses. Boca Raton: 2a ed. CRC Press Inc. 655p.

GASTON, K.J. \& BLACKBURN, T.M.. 1995. The frequency distribution of bird body weights: aquatic and terrestrial species. Ibis, 137:237-240.

GILL, F.B.. 1995. Ornithology. New York: W.H. Freeman and Company.

GINN, H.B. \& MELVILLE, D.S.. 1983. Moult in birds. British Trust for Ornithology, Tring Field Guide Hertfordshire, 19: 16-21.

GINN, H.B. \& MELVILLE, D.S.. 1995. Moult in birds. British Trust for Ornithology, guide 19. Norwich: Crowes of Norwich.

GOLDSMITH, F.B.. 1991. Monitoring for conservation and ecology. London, Chapman and Hall, 276p.

HARRINGTON, B.A. \& MORRISON, R.I.G.. 1982. Morphometric variation and habitat use of semipalmated sandpipers during a migratory stopover. Journal Field of Ornitholology, 53:258-262.

HARRINGTON, B.A. \& MORRISON, RI.G.. 1979. Semipalmated Sandpiper (Calidris pusilla). Wader Study Group Bulletin, 33: 37-38.

KARR, J.R.. 1979. On the use of the mist nets in the study of bird communities. Inland Bird Banding, Lawrence, 51(1):1-9.

KARSPRYK, M.J. \& HARRINGTON, B.A.. 1989. Manual de campo para maçaricos e batuíras. Seminário Internacional sobre manejo e conservação de maçaricos e ambientes aquáticos nas Américas. Itamaracá, Manomet bird observatory, $121 \mathrm{p}$.
LEGAGNEUX, P.; CLARK, R.G.; GUILLEMAIN, M.; ERAUD, C.; THÉRY, M.; BRETAGNOLLE, V. 2012. Large-scale geographic variation in iridescent structural ornaments of a long-distance migratory bird.Journal of Avian Biology Volume 43, Issue 4, pages 355-361.

MASCHER, J.W.. 1966. Weight variations in resting Dunlins (Calidris a. alpina) on autumn migration in Sweden. Bird Banding, 37:1-34.

MATTHIESSEN, P.. 1967. The Shorebirds of North America. New York, The Viking Press, 270p.

MORRISON, R.I.G.; ROSS, R.K.; ANTAS, P.T.Z. Brazil. In: MORRISON, R. I. G.; ROSS, R. K.. 1989. Atlas of neartic shorebirds on the coast of South America. Ottawa: Canadian Wildlife Service. 2:178-211.

NACINOVIC, J.. 2005. Aves marinhas na Bacia de Campos. Escola Nacional de Saúde Pública. FIOCRUZ. 60 pp.

NASCIMENTO, J.L.X.. 1998. Muda de Charadriidae e Scolopacidae (Charadriiformes) no Norte do Brasil. Ararajuba, 6: 141-144.

NOVELLI, R.. 1997. Aves Marinhas Costeiras do Brasil, Identificação e Biologia. Ed. Cinco Continentes. Porto Alegre.

SANTOS, T.R.S. \& ALVES, M.A.S.. 2011. Regis do Brasil, Identificação e Biologia. Ed. Cinco Continentes. Porto Aleet al. (Eds.). Conservacao de aves migratorias nearticas no Brasil. Belem: Conservacao Internacional. 406 pp.

SCHULZ-NETO, A.; SERRANO, I.L. \& EFE, M.A.. 2008. Muda e parâmetros biométricos de aves migratórias no norte do Brasil. Revista Ornitologia 3, Agosto de 2008. CEMAVE-ICMBio.

SENNER, S. \& HOWE, M.A.. 1984. Conservation of neartic shorebirds, p. 379-421. Behavior of marine animals, 5. Shorebirds: breeding behavior and populations. Plenum Press. New York.

SICK, H.. 1983. Migrações de aves na América do Sul continental. Publicação Técnica n.2. Brasília: Instituto Brasileiro de Desenvolvimento Florestal. 86p.

SICK, H.. 1997. Ornitologia Brasileira. Rio de Janeiro: Ed. Nova Fronteira.

TAVARES, D.V. \& SICILIANO, S.. 2014. Variação temporal na abundancia de espécies aquáticas em uma lagoa costeira do norte Fluminense, sudeste do Brasil. Biotemas 27(1): 121-132. 